2015-10

\title{
Social networks in changing
} environments

\author{
Wilson, Alexander
}

http://hdl.handle.net/10026.1/11442

10.1007/s00265-015-1973-2

Behavioral Ecology and Sociobiology

Springer Science and Business Media LLC

All content in PEARL is protected by copyright law. Author manuscripts are made available in accordance with publisher policies. Please cite only the published version using the details provided on the item record or document. In the absence of an open licence (e.g. Creative Commons), permissions for further reuse of content should be sought from the publisher or author. 


\title{
Social networks in changing environments
}

\author{
A. D. M. Wilson ${ }^{1,5}$ (I) S. Krause ${ }^{2}$ I. W. Ramnarine ${ }^{3}$ K. K. Borner ${ }^{1,4}$. \\ R. J. G. Clément ${ }^{1,4}$ • R. H. J. M. Kurvers ${ }^{1}$ • J. Krause ${ }^{1,4}$
}

Received: 5 June 2015 / Revised: 9 July 2015 / Accepted: 11 July 2015 / Published online: 26 July 2015

(C) Springer-Verlag Berlin Heidelberg 2015

\begin{abstract}
Social network analysis (SNA) has become a widespread tool for the study of animal social organisation. However despite this broad applicability, SNA is currently limited by both an overly strong focus on pattern analysis as well as a lack of dynamic interaction models. Here, we use a dynamic modelling approach that can capture the responses of social networks to changing environments. Using the guppy, Poecilia reticulata, we identified the general properties of the social dynamics underlying fish social networks and found that they are highly robust to differences in population density and habitat changes. Movement simulations showed that this robustness could buffer changes in transmission processes over a surprisingly large density range. These simulation results suggest that the ability of social systems to self-stabilise could have important implications for the spread of infectious
\end{abstract}

A. D. M. Wilson and S. Krause contributed equally

Communicated by J. Lindström

Electronic supplementary material The online version of this article (doi:10.1007/s00265-015-1973-2) contains supplementary material, which is available to authorized users.

A. D. M. Wilson

alexander.wilson@ymail.com

1 Department of the Biology and Ecology of Fishes, Leibniz Institute of Freshwater Ecology and Inland Fisheries, 12587 Berlin, Germany

2 Department of Electrical Engineering and Computer Science, Lübeck University of Applied Sciences, 23562 Lübeck, Germany

3 Department of Life Sciences, University of the West Indies, St Augustine, Trinidad and Tobago

4 Faculty of Life Sciences, Humboldt-Universität zu Berlin, Invalidenstrasse 42, 10115 Berlin, Germany

5 Centre for Integrative Ecology, Deakin University, 75 Pigdons Road, Waurn Ponds, VIC 3216, Australia diseases and information. In contrast to habitat manipulations, social manipulations (e.g. change of sex ratios) produced strong, but short-lived, changes in network dynamics. Lastly, we discuss how the evolution of the observed social dynamics might be linked to predator attack strategies. We argue that guppy social networks are an emergent property of social dynamics resulting from predator-prey co-evolution. Our study highlights the need to develop dynamic models of social networks in connection with an evolutionary framework.

Keywords Social dynamics · Network analysis · Population translocation $\cdot$ Habitat manipulation $\cdot$ Environmental change

\section{Introduction}

The social network approach (SNA) has provided us with powerful tools to address different aspects of an animal's social organisation because it provides many metrics to describe social patterns (Croft et al. 2008; Krause et al. 2009; Kurvers et al. 2014; Newman 2003). However, some social patterns have been shown to be highly dependent on environmental conditions (Darden et al. 2009; Henzi et al. 2009; Jacoby et al. 2010; Wey et al. 2013). Therefore, a potential short-coming of SNA is that these network metrics are largely just pattern descriptors which provide little insight into underlying processes that generate patterns and the robustness of these processes to environmental instability. An understanding of how social patterns emerge from interactions is particularly crucial for making testable predictions regarding changes in social network structure in response to environmental conditions or population composition. An important step in this direction was taken by Wittemyer et al. (2005) who investigated the change of social network patterns in a population of elephants over different seasons and by Flack et al. 
(2006) who used SNA to correctly predict fission processes in a primate group. Further progress has been made by using longitudinal network models to analyse changes in social network structure over time (Pinter-Wollman et al. 2013; Tranmer et al. 2015). Longitudinal models, however, often involve the aggregation of data on individual interactions over time and thus neglect the time-ordered nature of these interactions, which can have important repercussions for transmission processes (Blonder et al. 2012; Tranmer et al. 2015). Relational event models (REM) have been developed in recent years to account for such time-ordered interaction sequences (Butts 2008) and provide an interesting approach for modelling dynamic interactions. An alternative way of dealing with dynamic systems is to assess the state that an individual is in at regular time intervals and to identify the probabilities of state changes based on Markov chains (Wilson et al. 2014). Analogous to the long-established techniques of event- and point sampling (Martin and Bateson 2007), different aspects of social network dynamics can be recorded by REMs or Markov-based models. Biologically significant behaviours of short duration (e.g. aggression, mating) are better assessed with REMs whereas fission-fusion dynamics of social systems are more readily captured by Markov-based models.

Once key components of the social dynamics of individuals have been correctly identified, social networks become an emergent property of such Markov models and thus an output. This is important because such dynamic models allow deeper insights into the processes underlying social pattern formation and can generate social networks for quantitative predictions (Wilson et al. 2014). Here, we use the model by Wilson et al. (2014) in a way that allows a more detailed characterisation of the social dynamics (see Borner et al. 2015) to investigate the influence of experimental manipulations of the habitat and the social environment on social networks using replicated populations of guppies, Poecilia reticulata, in the wild.

Density is known to be an important predictor of social organisation (Krause and Ruxton 2002) but often neglected in the study of social networks. The size of groups (and in some cases also the number of groups) has been reported to increase with increased density under both lab and field conditions (Rangeley and Kramer 1998; Hensor et al. 2005; Makris et al. 2009; Strier and Mendes 2012; Brierley 2014). Increased densities result in higher encounter frequencies between individuals (Hensor et al. 2005), which in turn have consequences for social structure. In this study, we used our dynamic modelling approach (see above) to test the effects of changes in density (i.e. total number of fish divided by the total area) on social dynamics. We predict that if the density of fish per unit area goes up, the encounter rate should increase, and the time spent social should increase (and vice versa for a decrease in density). Density-modulated changes in social patterns are important because they could play a crucial role in processes of information transfer and disease transmission (Nightingale et al. 2014; Drewe and Perkins 2014). We explored this possiblity by modelling what impact the observed social dynamics of fish populations (undergoing density-changes) might have on transmission processes.

In addition to environmental density manipulations (the size of the pond and/or water depth), we also carried out social manipulations by removing all the males or all the females from a social network. Previous work carried out in captivity suggests that male presence disrupts connections between females (Darden et al. 2009). We investigated this prediction in the wild and also looked at male networks in the presence and absence of females to study the role of the latter in structuring male networks.

\section{Methods}

We caught and individually marked, using fluorescent elastomer tags (Northwest Marine Inc), all adult guppies (P. reticulata) in selected pools (Table 1) of the upper Turure river, Trinidad. Experimental work was carried out during the dry season in March and April 2011-2013 when water levels are low and guppies move relatively little between pools (Griffiths and Magurran 1997). Following marking, all individuals were released into their home pool and given $24 \mathrm{~h}$ to acclimate prior to the beginning of behavioural observations. We selected the pools on the basis of whether they provided good conditions for pool-side observations of ego-centric networks (see below). Pools 1a and $1 \mathrm{~b}$ were the same pool but in two different years (2012 and 2013; Table 1). However, due to annual flooding events and the short life span of guppies in general, no tagged fish from previous year's research were ever observed in consecutive years.

\section{Quantifying behavioural variables in the wild}

For each of the pools, we recorded the interaction dynamics of the fish by following a given marked focal fish for $2 \mathrm{~min}$ and recording the identity of its nearest neighbour every $10 \mathrm{~s}$. If no conspecific was present within four body lengths of the focal fish, the focal fish was regarded as having no neighbour for that observation point. Previous research has shown that this observation frequency/time interval provides ample opportunities for switching partners (Wilson et al. 2014). Upon completion of a 2-min observation period another marked fish was immediately chosen as a focal fish. This process was repeated consecutively until all individuals in a given pool had been observed and associations recorded for that observation session. Following completion of an entire observation session, the pool was left undisturbed for 10 min prior to beginning data collection for another session. This waiting period was chosen to insure that subsequent observation sessions were independent of the previous session (see Wilson et al. 2014). 
Table 1 Descriptive details for environmental and experimental aspects of all study pools

\begin{tabular}{|c|c|c|c|c|c|c|c|c|}
\hline Pool & Year & No. of fish (male/female) & Observation days & Treatment & Area & Density & Volume & Location \\
\hline \multirow[t]{2}{*}{$1 \mathrm{a}$} & \multirow[t]{2}{*}{2012} & \multirow[t]{2}{*}{$18(8 / 10)$} & 3 & UM & $16.42 \mathrm{~m}^{2}$ & 1.10 & $3.30 \mathrm{~m}^{3}$ & \multirow{3}{*}{$\begin{array}{l}10^{\circ} 41^{\prime} 06.5^{\prime \prime} \mathrm{N} \\
61^{\circ} 10^{\prime} 23.4^{\prime \prime} \mathrm{W}\end{array}$} \\
\hline & & & 3 & SM & & & & \\
\hline 4 & & & 3 & $\mathrm{TL}$ & $8.04 \mathrm{~m}^{2}$ & 2.24 & $1.64 \mathrm{~m}^{3}$ & \\
\hline \multirow[t]{3}{*}{$1 b$} & \multirow[t]{3}{*}{2013} & \multirow[t]{3}{*}{$14(5 / 9)$} & 2 & UM & $14.45 \mathrm{~m}^{2}$ & 0.97 & $2.83 \mathrm{~m}^{3}$ & \multirow{3}{*}{$\begin{array}{l}10^{\circ} 41^{\prime} 06.5^{\prime \prime} \mathrm{N} \\
61^{\circ} 10^{\prime} 23.4^{\prime \prime} \mathrm{W}\end{array}$} \\
\hline & & & 2 & HWL & $22.70 \mathrm{~m}^{2}$ & 0.62 & $5.24 \mathrm{~m}^{3}$ & \\
\hline & & & 2 & LWL & $11.16 \mathrm{~m}^{2}$ & 1.25 & $2.03 \mathrm{~m}^{3}$ & \\
\hline \multirow[t]{3}{*}{2} & \multirow[t]{3}{*}{2013} & \multirow[t]{3}{*}{$11(3 / 8)$} & 2 & UM & $2.65 \mathrm{~m}^{2}$ & 4.15 & $0.48 \mathrm{~m}^{3}$ & $10^{\circ} 41^{\prime} 8^{\prime \prime} \mathrm{N}$ \\
\hline & & & 2 & HWL & $3.46 \mathrm{~m}^{2}$ & 3.18 & $0.93 \mathrm{~m}^{3}$ & \multirow{3}{*}{$61^{\circ} 10^{\prime} 22^{\prime \prime} \mathrm{W}$} \\
\hline & & & 2 & LWL & $1.17 \mathrm{~m}^{2}$ & 9.40 & $0.14 \mathrm{~m}^{3}$ & \\
\hline 3 & \multirow{4}{*}{2013} & \multirow{4}{*}{$8(4 / 4)$} & 2 & $\mathrm{TL}$ & $6.84 \mathrm{~m}^{2}$ & 1.61 & $1.23 \mathrm{~m}^{3}$ & \\
\hline \multirow[t]{3}{*}{3} & & & 2 & UM & $6.84 \mathrm{~m}^{2}$ & 1.17 & $1.23 \mathrm{~m}^{3}$ & \multirow{4}{*}{$\begin{array}{l}10^{\circ} 41^{\prime} 8^{\prime \prime} \mathrm{N} \\
61^{\circ} 10^{\prime} 22^{\prime \prime} \mathrm{W}\end{array}$} \\
\hline & & & 2 & HWL & $8.20 \mathrm{~m}^{2}$ & 0.98 & $1.80 \mathrm{~m}^{3}$ & \\
\hline & & & 2 & LWL & $4.55 \mathrm{~m}^{2}$ & 1.76 & $0.66 \mathrm{~m}^{3}$ & \\
\hline 2 & \multirow{3}{*}{2012} & \multirow{3}{*}{$22(11 / 11)$} & 2 & $\mathrm{TL}$ & $2.65 \mathrm{~m}^{2}$ & 3.02 & $0.48 \mathrm{~m}^{3}$ & \\
\hline \multirow[t]{2}{*}{5} & & & \multirow[t]{2}{*}{$2+2$} & \multirow[t]{2}{*}{ SM } & \multirow[t]{2}{*}{$47.5 \mathrm{~m}^{2}$} & \multirow[t]{2}{*}{0.46} & \multirow[t]{2}{*}{$9.5^{\mathrm{a}} \mathrm{m}^{3}$} & $10^{\circ} 40^{\prime} 51.5^{\prime \prime} \mathrm{N}$ \\
\hline & & & & & & & & $61^{\circ} 10^{\prime} 4.5^{\prime \prime} \mathrm{W}$ \\
\hline
\end{tabular}

For the translocation experiments, the identity of the new pool that the fish were moved to is given. Treatments: UM (unmanipulated), TL (translocation to a different pool), HWL (high water level), LWL (low water level), and SM (sex manipulations)

${ }^{a}$ No water depth measurements were taken for pool 5 and the values presented are estimates based on typical average depths of other pools

This process was repeated five to six times per day for each pool, occurring between 09:00 and 14:00. The number of observation days for each pool is given in Table 1 .

\section{Experimental manipulations of pools}

\section{Environmental manipulations: water levels changes and translocations}

Experimental manipulations of water levels were carried out in pools $1 \mathrm{~b}, 2$, and 3 and for translocation experiments we used the fish in pools 1a, 2, and 3 (see Table 1). First, all interactions between fish in unmanipulated pools were recorded (see Table 1 for observations periods). Second, and depending on the pool, up to three consecutive experimental manipulations were conducted on a given pool as represented by (1) an increase in area from original water level, (2) a decrease in area from original water level, and (3) translocation of all marked guppies from their original pool to a nearby similar, but isolated (guppy-free) pool.

To change fish density, manipulations of pool water column height were carried out by adding or removing rock substrate from the stream inflow or outflow, respectively, in the observation pool. Such changes in water column depth occur regularly in natural habitats as a result of strong rainfalls within and between seasons and are likely to result in significant changes to the fish density but can also affect other environmental parameters of the observation pool (e.g. water flow, food distribution, population density, and refuge availability). For the purposes of this experiment, only pool surface area and water depth were quantified in detail. The manipulations of the water level resulted in variable changes in surface area and volume between pools (Table 1). This process could not be entirely standardised under field conditions because the natural features of pools had to be taken into account, which constrained our options regarding such manipulations.

Following the above environmental manipulations, all marked individuals (in pools 1a, 2, and 3) were then caught using dipnets and transported to another isolated pool within $20 \mathrm{~m}$ up- or downstream. Support capacity of the novel pool was determined by the a priori presence of a resident guppy population prior to removal. Fish from pool 1a were translocated into a new pool (pool 4) slightly downstream and fish from pools 2 and 3 were translocated into each other's pools (see unmanipulated dimensions, Table 1). After the experiments were finished all translocated fish were returned to their point of origin.

\section{Sex manipulations}

On day 1, we first removed all 11 males that were present in pool 5 (by carefully targeted selection using dipnets) and recorded three observation sessions (see method above) with 11 females only (Table 1). We then returned the males and recorded the female association patterns in the presence of males for another three observation sessions. However, in this case we ignored males when they were the nearest neighbour of a female focus fish. Thus we recorded female-female interactions in the presence and absence of males and always focussed on the females. On day 2, we reversed our protocol 
by initially recording female networks in the presence of males and then secondly, in the absence of males for three observation sessions respectively, thus controlling for treatment order. As an additional control, we also disturbed the pool in the morning of the $2^{\text {nd }}$ day by simulating the capture of males (without actually removing any individuals) as had occurred on the previous morning of day 1 .

On days 3 and 4, we carried out the same procedure as above but in this instance we removed all 11 females from pool 5 to investigate male-male interactions in the presence and absence of females. In this case, females were ignored when recording nearest neighbour associations regardless of their presence once returned post-removal.

We also examined the impact of the addition of five new females to pool 1a over a three day period (after an observation period of 3 days of the unmanipulated pool). These females were removed from this pool before the translocation of the fish to pool 4.

\section{Analysis}

\section{Modelling of social dynamics}

To describe the underlying social dynamics of the fissionfusion behaviour of the observed fish and to investigate potential differences between the pool-populations and treatments we used the fission-fusion model by Wilson et al. 2014, which is based on Markov chains. It describes the social behaviour common to all focal individuals as sequences of 'behavioural states' (Fig. 1). In the presence of $k$ potential neighbours at each time point, a focal fish can either be with a nearest neighbour $g, 1 \leq g \leq k$, denoted by $s_{g}$ or alone (no conspecific within 4 body lengths) denoted by $a$. By regarding $a$ and all $s_{g}$ as states of a first-order Markov chain, the transition probabilities between these states can be estimated from the data points in our observations (see Supplementary material or Wilson et al. 2014 for more details). Following Wilson et al. 2014 we did not take the specific individual identities into account when estimating the model probabilities. This means, our model describes the general dynamics common to all fish.

A focal fish is regarded as being social, if it is in state $s_{g}$ for some neighbour $g$. 'Being social' is not an explicit state in the model, but is implicitly defined by the set of states $s_{1} \ldots s_{k}$. The model can be characterised by specifying the probabilities of leaving the current nearest neighbour ( $\left.p_{\text {leave nn }}\right)$, of ending social contact in general $\left(p_{s \rightarrow a}\right)$, and of ending being alone $\left(p_{a \rightarrow s}\right)$. The reciprocal values of theses probabilities determine the mean number of data points over which a focal fish will retain the state of being next to a specific neighbour, of being social, and of being alone, respectively. Multiplying this number by $10 \mathrm{~s}$ (the time between two data points) yields the mean time spent in each state. In our investigations, we also looked at the probability of changing the nearest neighbour while staying social $p_{\text {switch }}=p_{\text {leave } \_n n}-p_{s \rightarrow a}$ to better illustrate the behaviour of the guppies.

It has been shown that this model can be used to describe the social dynamics of female guppies in the wild (Wilson et al. 2014) and of mixed-sex groups of guppies in the lab (Borner et al. 2015). Figure S1 in the supplementary material demonstrates this for our pools by showing that the observed lengths of social contact, of contact with a particular nearest neighbour, and of being alone are well approximated by the model predictions.

The model probabilities are estimated as simple proportions (see supplementary material or Borner et al. 2015 for more details). We compared them by looking at their $95 \%$ confidence intervals that were computed using the function prop.test in R. This allows us to investigate potential differences between the pools or between the treatments regarding the lengths of social contact phases, of contact phases with the same neighbour, and of being alone, where the length is measured as multiples of time intervals of $10 \mathrm{~s}$.

\section{Influence of density changes}

When densities are manipulated, we should observe changes in the values of the model probabilities $p_{s \rightarrow a}, p_{a \rightarrow s}$, and $p_{\text {switch nn. }}$. For example, if the density decreases, the encounter probabilities between fish will also decrease. As a consequence, the lengths of phases of being alone will increase. Similarly, after leaving the current neighbour there will be a higher chance of being alone. In terms of the model probabilities, this means that with decreasing density $p_{a \rightarrow s}$ and $p_{\text {switch_nn }}$ should also decrease. The time spent with a particular neighbour should not change very much, but $p_{s \rightarrow a}$ should increase as $p_{\text {switch_nn }}$ decreases because $p_{\text {retain_nn }}+p_{s \rightarrow a}+p_{\text {switch_nn }}=1$ (Fig. 1), i.e. the lengths of phases of being social will decrease.

To roughly estimate the expected magnitude of these changes, we performed simulations of random movements of individuals, where each individual followed the same simple rules (see supplementary material for a detailed description of the movement simulations). The influence of density changes on social structure has already been investigated (Hensor et al. 2005). However, here we need to simulate a process that changes the behavioural states of the individuals in order to estimate our Markov chain model probabilities.

We examined a wide range of parameter settings of the movement simulations and found that $p_{a \rightarrow s}$ and $p_{\text {switch nn }}$ linearly decreased with decreasing density. The parameters of the linear function depended on the parameter settings of the movement simulations. This means, we cannot draw conclusions about absolute differences of $p_{a \rightarrow s}$ and $p_{\text {switch_nn. There- }}$ fore we chose one pool and treatment (pool 3 before manipulations) as a reference point and determined the expectations for other pools and treatments relative to it. We restricted this 


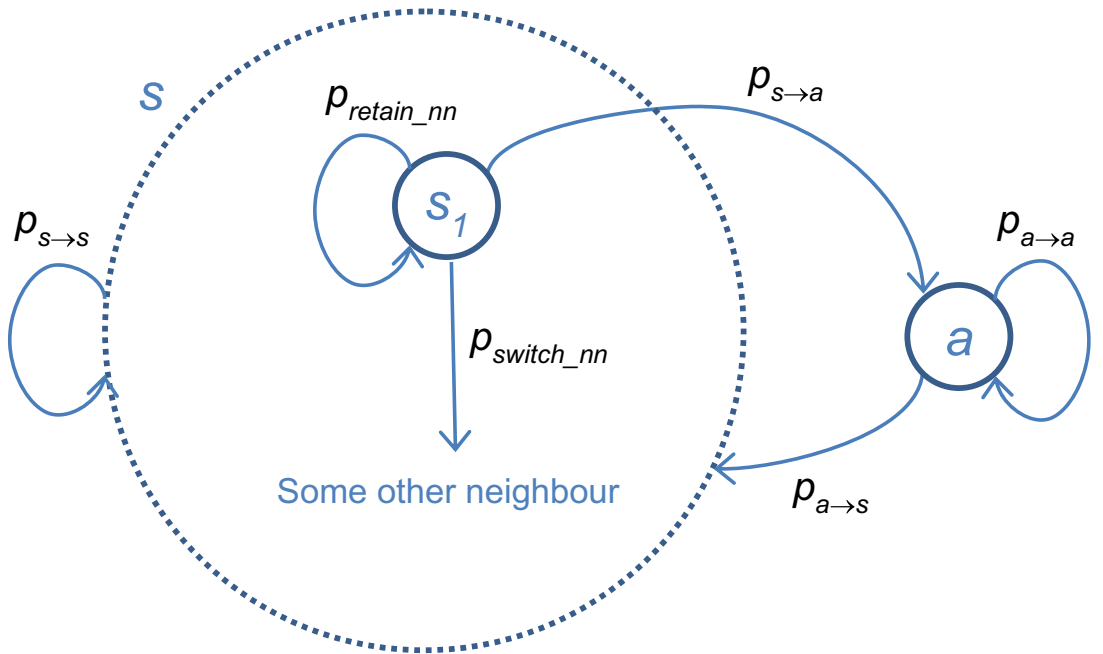

Fig. 1 Markov chain model of the fission-fusion behaviour in the guppy where a focal fish can either be with a nearest neighbour $g$ denoted by $s_{g}$ or alone (no conspecific within four body lengths) denoted by $a$. It stays with its current nearest neighbour with probability $p_{\text {retain nn. }}$. When the contact with this neighbour ends it decides to be alone with probability $p_{s \rightarrow a}$ or switches to a different neighbour with probability $p_{\text {switch nn. In }}$ our model, the dynamics do not depend on the neighbour's identity and is the same for each state $s_{g}$. Therefore, for the sake of clarity the figure only

investigation to the translocation experiments (pools 1a, 2, and 3) because here the biggest changes in density occurred.

We proceeded as follows. In a first step, we chose the parameters of the movement simulation such that it roughly reproduced the values of $p_{\text {leave_nn, }}, p_{s \rightarrow a}, p_{a \rightarrow s}$, and $p_{\text {switch_nn }}$ that we observed at pool 3 before manipulations. In a second step, we applied the movement simulation to other pools and translocation treatments taking into account the numbers of individuals and the respective areas (by choosing the radius of the simulation world accordingly). To get results that are comparable with our real observation, we "observed" the simulations using the same observation scheme as for the real observations (i.e. same number of sessions and same number of time points per focal individual). We repeated the second step 1000 times for each pool and translocation treatment.

\section{Transmission processes}

Changes in the social dynamics can have important consequences for transmission processes (Krause et al. 2014). For example, the transmission of a disease might depend on the time two individuals are close to each other. This means, even if the total amount of time being social does not change, transmission processes can potentially be affected by changes in the fine structure of the social dynamics (Wilson et al. 2014). To demonstrate potential consequences for our study system, we used our Markov chain model to simulate transmission processes for different settings of $p_{\text {leave nn }}, p_{s \rightarrow a}, p_{a \rightarrow s}$, and $p_{\text {switch nn }}$ for pool 2. We followed the approach of Wilson et al. (2014) and generated a sequence of behavioural states shows the state $s_{l}$. If there are $k$ potential neighbours other than $s_{l}$, the probability of choosing a particular one is $p_{\text {switch nn }} / k$. The probability $p_{\text {leave_nn }}=1-p_{\text {retain_nn }}=p_{s \rightarrow a}+p_{\text {switch_nn }}$ is not explicitly shown in the figure. Its reciprocal value determines the mean length of contact with a specific neighbour. A focal fish is regarded as being social, denoted by $s$ (dotted circle), if it is in state $s_{g}$ for some neighbour $g$. It stays being social with probability $p_{s \rightarrow s}=1-p_{s \rightarrow a}$

for some focal individual in the presence of $k$ (10 in our case) potential nearest neighbours, e.g.

$a, a, s_{1}, s_{1}, s_{2}, s_{2}, s_{2}, a, a, a, a, a, s_{3}, s_{1}, a, \ldots$

Under the assumption that it takes $m$ consecutive time steps to transmit a disease from one individual to another we determined how many time steps it takes from the beginning of such a sequence until the focal individual has been involved in a contact phase of length $\geq m$ with an infected individual. For each setting of the probabilities, we repeated the simulation $10^{6}$ times and computed the mean time until infection. We investigated scenarios, where one out of 10 potential neighbours was infected and where $m$ had the values 3,4 , and 5 (which correspond to 30,40 , and $50 \mathrm{~s}$, respectively, on our timescale).

\section{Consistency of individual preferences}

An interesting aspect of social structure is social preferences for particular individuals (which in guppies is often connected to cooperative relationships; Croft et al. 2009). For our investigation, we used two different ways to quantify the tie strength of a pair of individuals' $i_{1}$ and $i_{2}$, (a) according to the number of contact phases between $i_{1}$ and $i_{2}$, and (b) according to the mean duration of contact between $i_{1}$ and $i_{2}$. We determined the tie strength for each pair of individuals for each treatment by computing the sum of these values (according to $a$ or $b$, respectively) across all sessions and observation days of this treatment. We investigated both ways of expressing the tie strength separately. 
In a first step for each pool, we analysed individual preferences for each treatment separately by using a randomisation test where for each focal individual we kept constant the number of observed contact phases as well as their lengths and randomly assigned the identities of its neighbours. As a test statistic, we used the sum of squares of the tie strengths of all pairs of individuals, which we computed in the two above described ways. In the absence of individual preferences (null hypothesis), the tie strengths should not differ very much between the pairs of individuals, yielding moderate values for the test statistic. If the observed value of the test statistic is extremely large (among the $5 \%$ largest values yielded by the randomisation procedure), this indicates that the observed tie strength cannot be explained by randomly chosen neighbours.
Given that individual preferences were detected in each treatment of each pool, we tested whether the preferences were consistent across the treatments for each pool. However, individual preferences were only detected in terms of the frequency of contact but not its duration (which is in accordance with the findings of Wilson et al. 2014). Therefore, in our consistency analysis we only used as tie strength the number of contact phases. To test whether the null hypothesis of no consistency is to be rejected, we randomised the identities of the individuals in each matrix of pairwise tie strengths (per treatment). For each pair of individuals, we computed the variance of their tie strengths across the treatments. The sum of these variances constituted our test statistic. Small values of this test statistic indicate similar individual preferences across the treatments and if
Fig. 2 The probabilities (plus $95 \%$ confidence intervals) of a ending contact with a specific neighbour ( $\left.p_{\text {leave_nn }}\right), \mathbf{b}$ ending social contact in general $\left(p_{s \rightarrow a}\right)$, c ending being alone $\left(p_{a \rightarrow s}\right)$, and $\mathbf{d}$ changing to a different social partner while staying social $\left(p_{\text {switch_nn }}\right)$ are shown for all pools before manipulation (see also Fig. 1 for the full Markov model) a

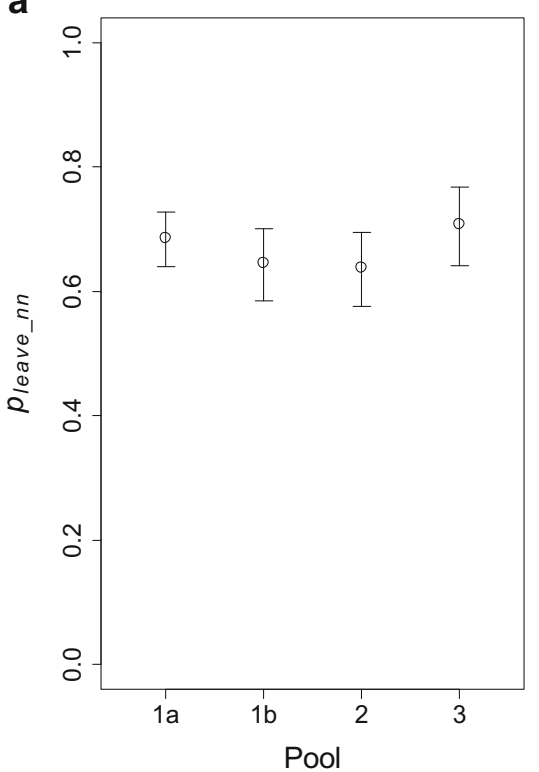

C

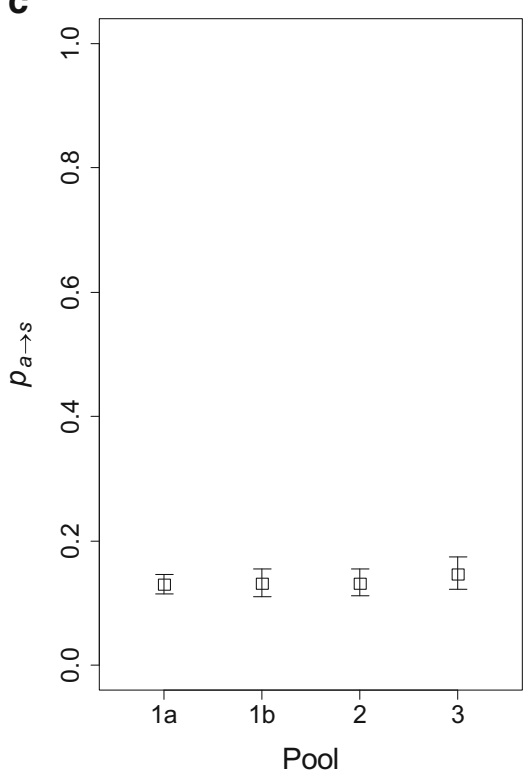

b

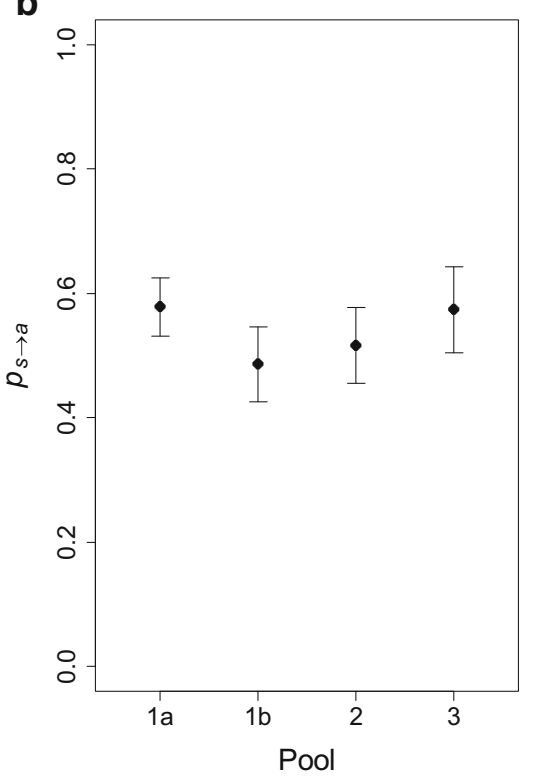

d

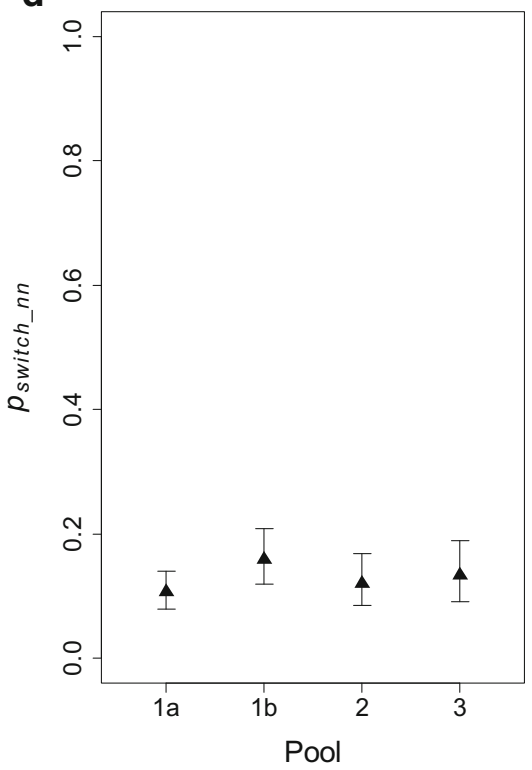


the observed value is among the $5 \%$ smallest values produced by the randomisation procedure, we can reject the null hypothesis. Some individuals did not occur in all sessions of a treatment and had therefore consistently small numbers of contact phases with all other individuals. To avoid spurious results caused by this, we used relative tie strengths which were computed by dividing the number of contact phases of each pair of individuals by the number of sessions in which both of them were present. This is based on the assumption that the number of contact phases is proportional to the number of sessions, which seems reasonable in our case. However, this procedure decreases the test power because rarely occurring individuals will have the same weight as frequently occurring ones.

To level the influence of the different numbers of contact phases in the treatments, we also performed the test with normalized numbers of contact phases where each absolute number was divided by the total number of contact phases per treatment. Figures 2, 3, 4, 5, 6, and 7 were plotted using $\mathrm{R}$ version 3.0.3.

\section{Results}

\section{Environmental density manipulations: water level changes and translocations}

The social dynamics of guppies was well approximated by a geometric distribution (Fig. S1) and was largely independent

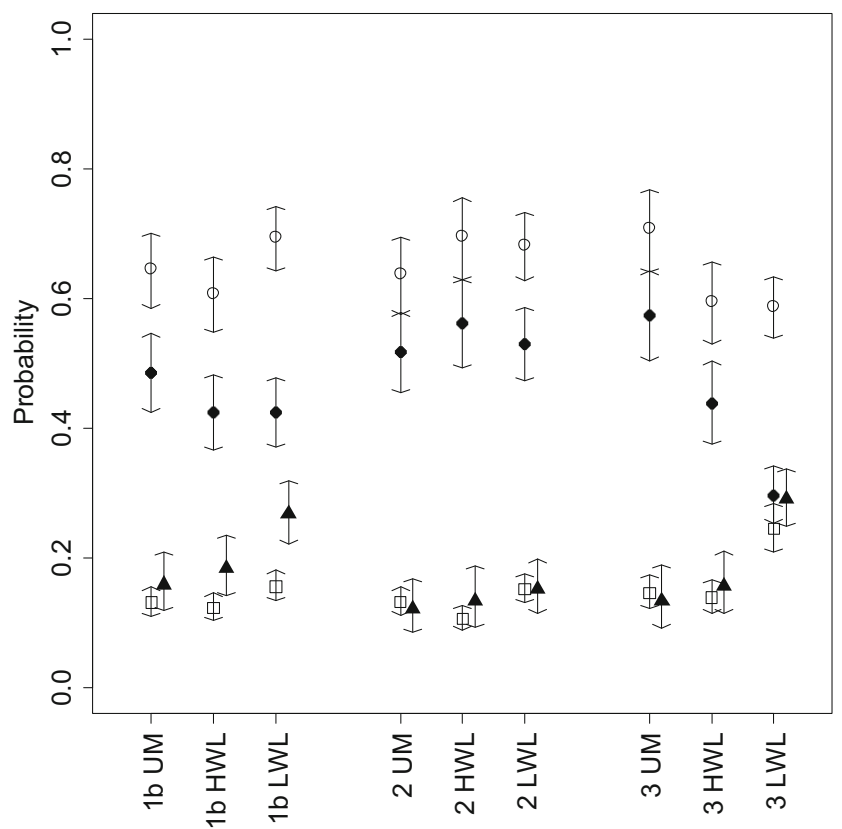

Fig. 3 The probabilities (plus $95 \%$ confidence intervals) of (open circles) ending contact with a specific neighbour ( $\left.p_{\text {leave_nn }}\right)$, (filled circles) ending social contact in general $\left(p_{s \rightarrow a}\right)$, (squares) ending being alone $\left(p_{a \rightarrow s}\right)$, and (filled triangles) changing to a different social partner while staying social ( $\left.p_{\text {switch nn }}\right)$ are shown for pools $1 \mathrm{~b}, 2$, and 3 for each of the water levels treatments unmanipulated, high water (HWL) and low water (LWL) (see also Fig. 1 for the full Markov model) of density and other environmental conditions. We found no major differences in the dynamics between different pools of the same or different years (Fig. 2) despite the fact that density (measured as fish per square metre) differed by up to 4.3 times between unmanipulated pools and sex ratios varied (0.38-1, males to females; Table 1).

Water level changes produced large changes in available surface area and hence density (up to 3-fold) but had relatively little impact on social dynamics (Fig. 3). The only exceptions are $p_{s \rightarrow a}$ and $p_{a \rightarrow s}$ in pool 3 during the low water treatment (Fig. 3). The most surprising result is probably the absence of changes in the fission-fusion dynamics after translocation to new pools while our simulations of random movements suggest that we should see considerable differences, in particular regarding $p_{a \rightarrow s}$ and $p_{\text {switch nn }}$ (Fig. 4). Fish were transferred into pools that varied between roughly half to double the surface area of their original pool and pools also differed considerably in shape. This suggests that the fish responded to these environmental changes by adapting their behaviour such that the patterns described by the Markov model (lengths of phases of contact and of being alone) are retained.

Figure 5 demonstrates potential consequences of such behaviour for transmission processes. In the case of the fish in pool 2 (which were translocated into the much larger pool 3), the fact that guppies maintain their fission-fusion dynamics (see results above) would result in disease transmission times that are shorter than predicted by our MC model (see methods regarding transmission processes).

\section{Individual social preferences}

We detected individual preferences in each treatment of each pool (listed in Table 1), if the tie strength was defined by the number of contact phases (all $p$ values $<0.016$, number of randomisation steps $=10^{4}$ ) but not if it was defined by the mean duration of contact (all $p$ values $>0.05$, number of randomisation steps $=10^{4}$ ). This confirms the findings of Wilson et al. (2014) and shows that in our study system the preference for particular social partners is not expressed in the length of associations but in their frequency. It also helps to explain why the lengths of social contact and of contact with a particular neighbour can be described by a model common to all guppies in a pool (Fig. 1). Thus, individuals do not vary in how often they change associates, but do differ in how likely they are to associate with particular individuals.

The individual preferences (in terms of numbers of contact phases) were consistent across the 4 treatments for pool $3(p<0.001)$, the four treatments for pool $2(p=0.05)$, and the 3 treatments for pool $1 \mathrm{~b}(p<0.001)$. However, we did not find consistency across the two treatments for pool 1a $(p=0.34)$. The number of randomisation steps was $10^{4}$ in each test. 
a

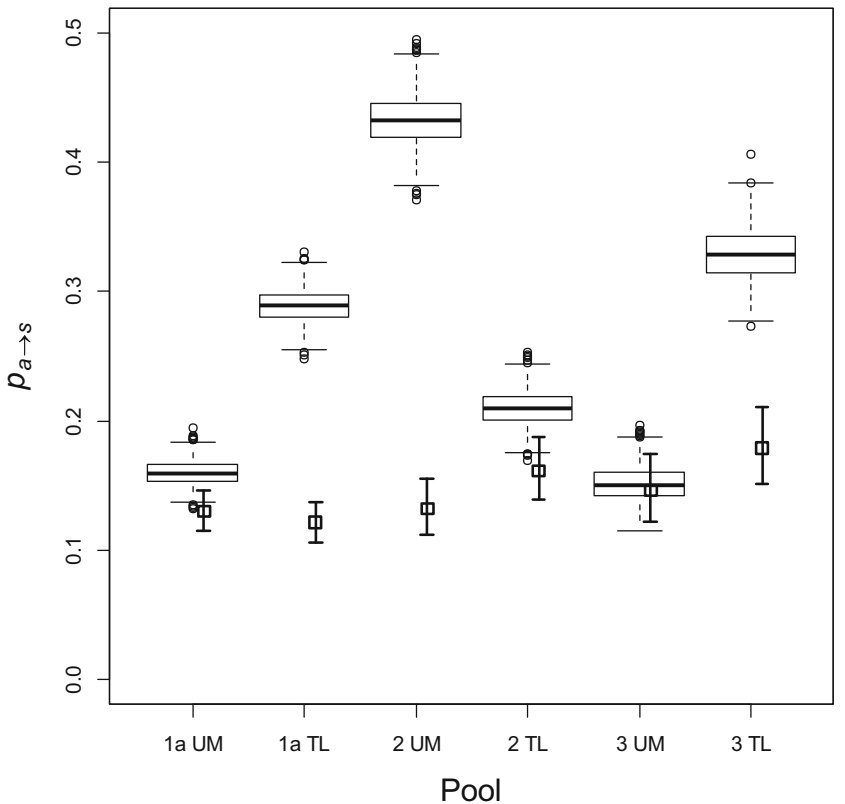

C

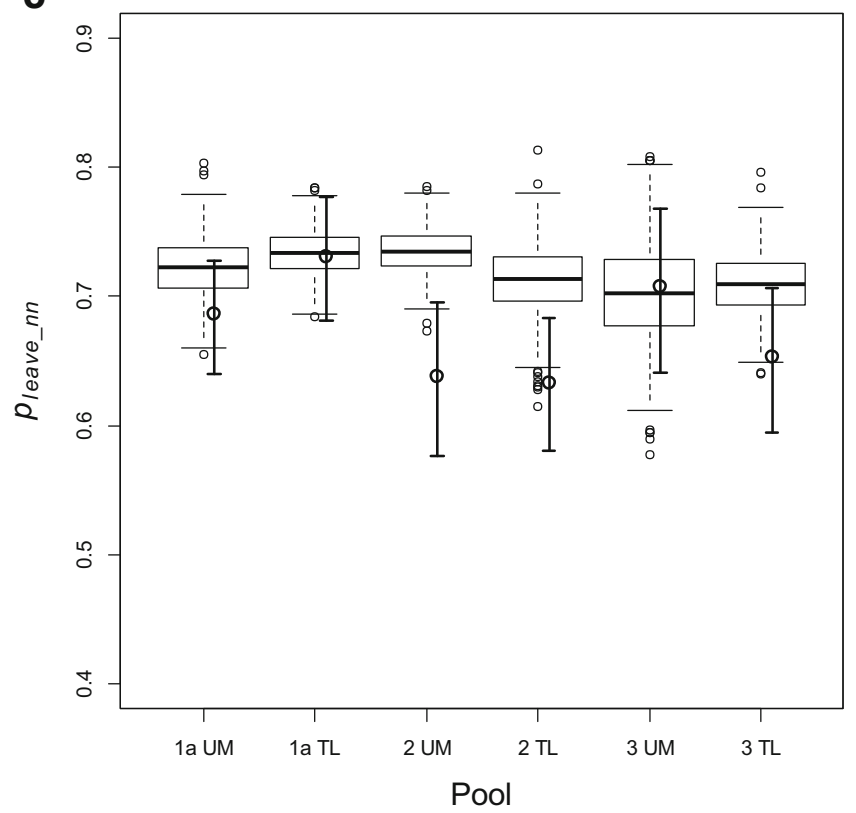

Fig. 4 Comparison of probabilities of a Markov chain model estimated from observations (with $95 \%$ confidence intervals) and from simulated movements (box-and-whisker plots) for pools 1a, 2, and 3 before manipulations (UM) and after translocation (TL). The box-and-whisker plots show median, quartiles, and the most extreme data points. The whiskers mark the most extreme data points that have a distance from the box of at most 1.5 times the length of the box. The parameters of the

\section{Social manipulations}

Female-female networks (in the presence of males) and male-male networks (in the presence of females) show very similar interaction dynamics (Fig. 6). This is an important prerequisite for all the work shown above in b

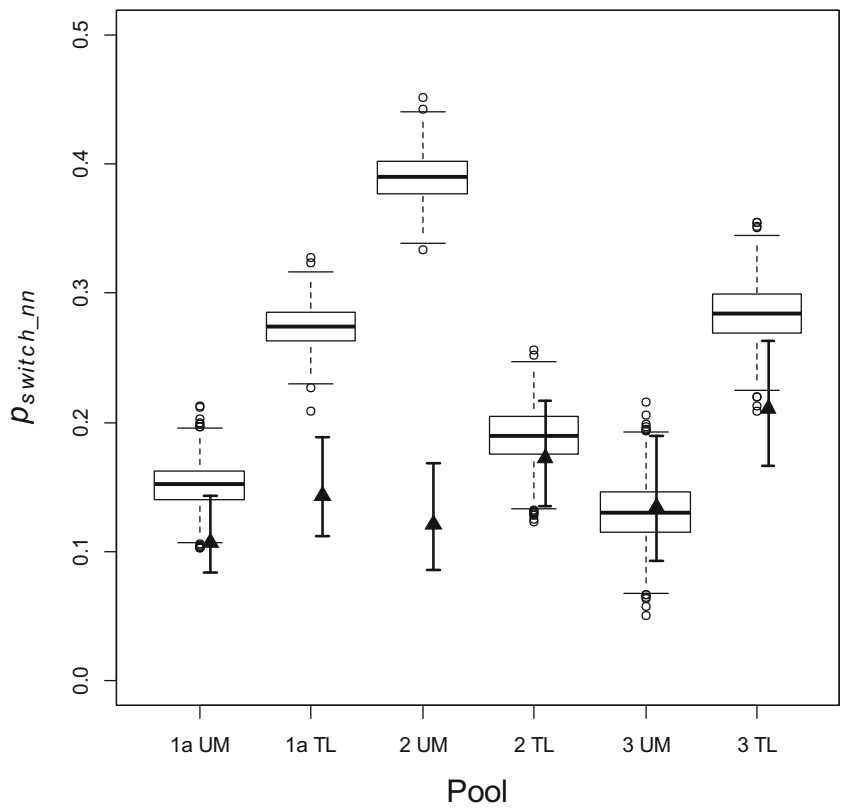

d

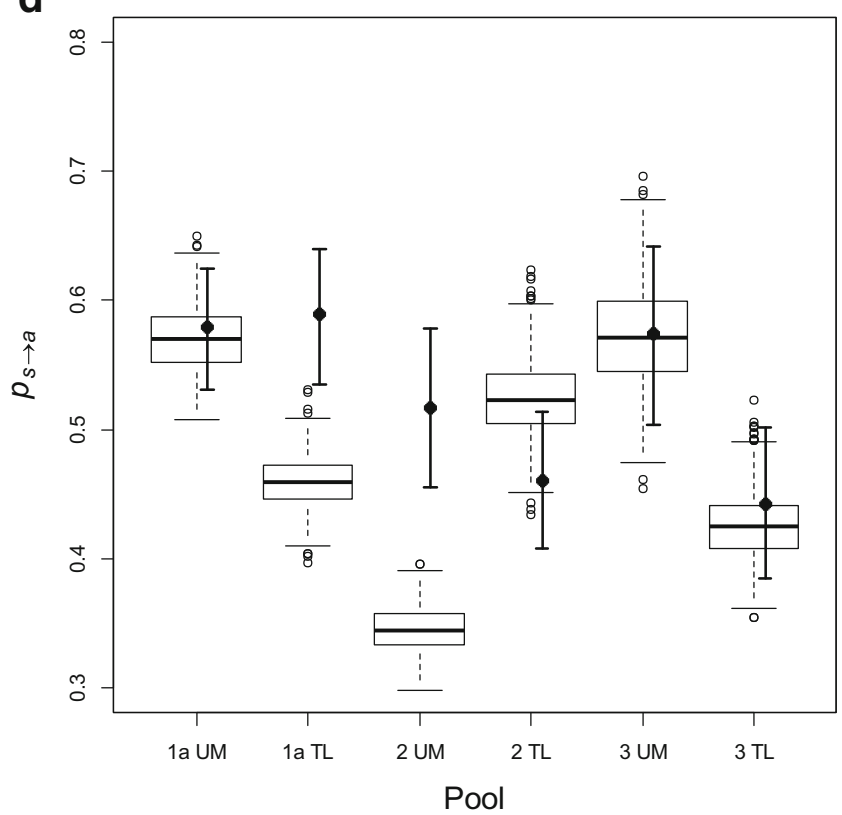

simulated movements were chosen such that the simulation roughly reproduced the probabilities of pool 3 before manipulation. The box plots show the results of 1,000 repetitions of the simulation. The figure contains the probabilities of a ending being alone $\left(p_{a \rightarrow s}\right)$, $\mathbf{b}$ changing to a different social partner while staying social $\left(p_{\text {switch nn }}\right)$, c ending contact with a specific neighbour $\left(p_{\text {leave_nn }}\right)$, and $\mathbf{d}$ ending social contact in general $\left(p_{s \rightarrow a}\right)$

which we did not carry out separate analyses for males and females.

Despite the fact that removal of males and females respectively, reduces fish density by $50 \%$ in both cases we see very different responses of males and females to this manipulation. Females greatly decrease the probability of ending their social 
Fig. 5 Mean number of time points until infection of a focal individual among 10 potential neighbours one of which is infected as a function of the length of contact necessary for the transmission of a disease as predicted by a simulation based on a Markov model for pool 2 a before manipulations and $\mathbf{b}$ after translocation into the larger pool 3. The parameters of the Markov model were estimated from the observations (filled circles) and from simulated random movements (open circles) that were parameterized, such that they roughly reproduced the Markov model probabilities before manipulations a

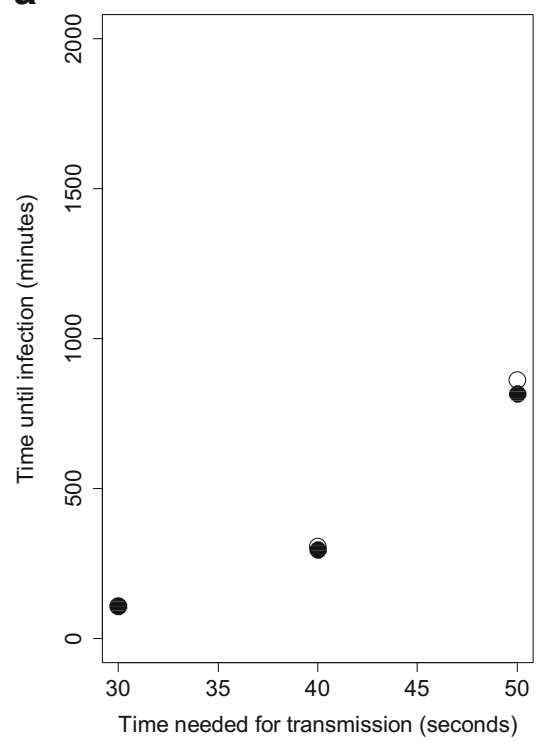

b

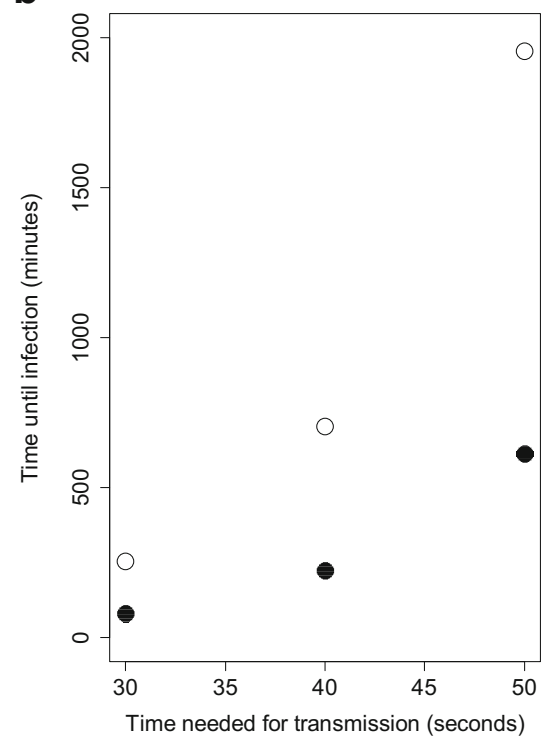

contact $\left(p_{s \rightarrow a}\right)$ and the probability of ending social contact with a specific neighbour ( $p_{\text {leave nn }}$ ) whereas males greatly increase these probabilities (Fig. 6). The probability of ending time alone $\left(p_{a \rightarrow s}\right)$, is affected in the opposite way by the manipulation in either sex, in particular for males (while the confidence intervals for females overlap). This means that females increase the total time being social.

We also found consistent preferences across the two femalefemale networks in the presence and the absence of males ( $p=$ 0.013 , number of randomisation steps $=10^{4}$ ). This means that, while the presence of males shortens the contact phases between females, it does not seem to affect female preferences.

The introduction of five new females into pool la resulted in a change of $p_{s \rightarrow a}$ and $p_{\text {leave_nn }}$ (the probabilities of ending social time and of ending time with a specific neighbour) on day 1 of the manipulation (Fig. 7). However, after the first day, we can see that this effect was diminished and by day three the dynamics were back to normal (Fig. 7).

\section{Discussion}

The social dynamics of guppies was remarkably consistent across different pools, sampling years, and even across environmental manipulations. Our findings suggest that there is a generic fission-fusion behaviour that guppies maintain in their social dynamics, though it might be specific to the Turure population. It also appears that there are aspects of social organisation that are unique to each social network (i.e. social preferences in each network), which are preserved across manipulations including translocations. In contrast to environmental manipulations, social manipulations caused significant changes in the social dynamics of guppy populations, with opposite effects in males and females.

\section{Environmental manipulations: water level changes and translocations}

Density increases are often accompanied by increases in absolute number of individuals making it difficult to separate out the effects of each (Rangeley and Kramer 1998; Hensor et al. 2005; Makris et al. 2009; Strier and Mendes 2012; Brierley 2014). In our study, density and total number of individuals were independent and we found that they had little effect on social dynamics. Thus the observed fission-fusion behaviour appeared to be density independent (within the range of parameters that we tested). Another surprising result was that fish maintained their social dynamics even when transferred into new and unfamiliar pools (resulting in decoupling of the social system from the habitat).

Our movement simulations show that the changes within and between pools should strongly affect encounter probabilities of fish and thereby social dynamics (see Hensor et al. 2005 for a null model of shoaling behaviour). Nevertheless, guppies maintained their fission-fusion dynamics, which is an indication that there is a strong selection pressure on guppies to work actively against any changes in their social dynamics. There are two different ways in which fish could achieve this consistency in their social dynamics. One option would be for fish that were transferred into a larger pool to utilize only a small portion of this pool and thereby maintain the same density as before. However, this was not observed (fish always used the entire pool). The other option is that fish adjust their swimming speeds (or the directedness of their swimming path when approaching conspecifics) to pool size; i.e. swimming faster in larger pools and slower in smaller ones. The latter is a prediction that can be tested by future studies. Our study shows that this consistency in 
Fig. 6 In pool 5, same-sex networks (males and females, respectively) were recorded in the presence and absence of the opposite sex. The probabilities (plus $95 \%$ confidence intervals) of a ending contact with a specific neighbour $\left(p_{\text {leave_nn }}\right), \mathbf{b}$ ending social contact in general $\left(p_{s \rightarrow a}\right)$, c ending being alone $\left(p_{a \rightarrow s}\right)$, and $\mathbf{d}$ changing to a different social partner while staying social $\left(p_{\text {switch_nn }}\right)$ are shown for all pools before manipulation (see also

Fig. 1 for the full Markov model) a

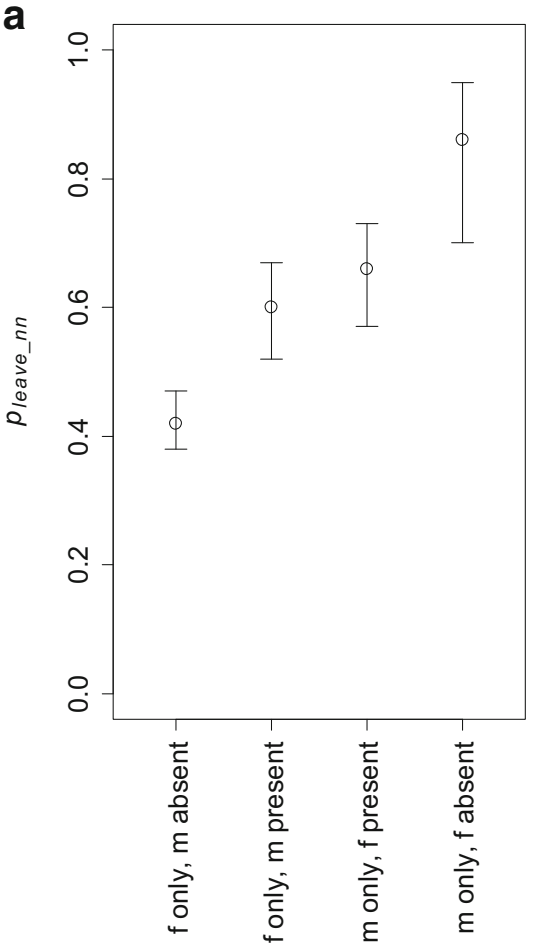

C

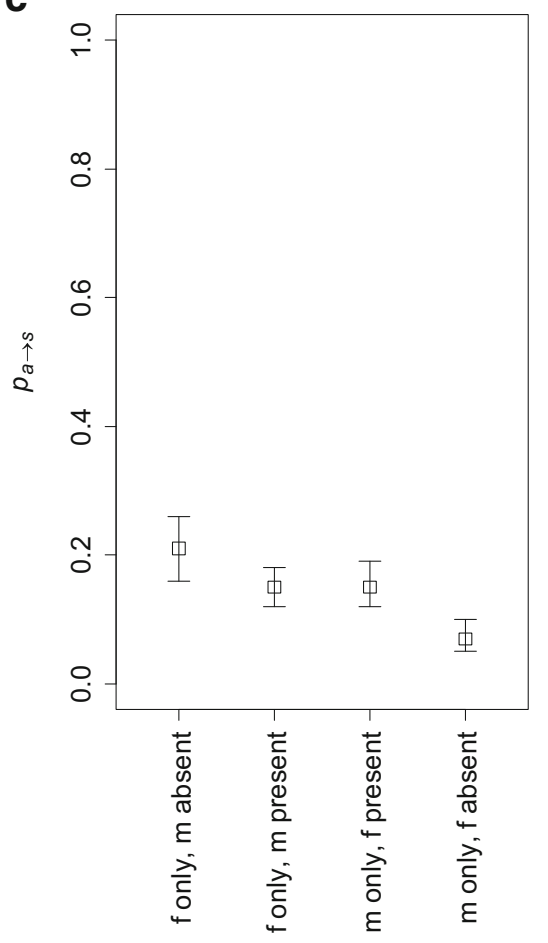

b

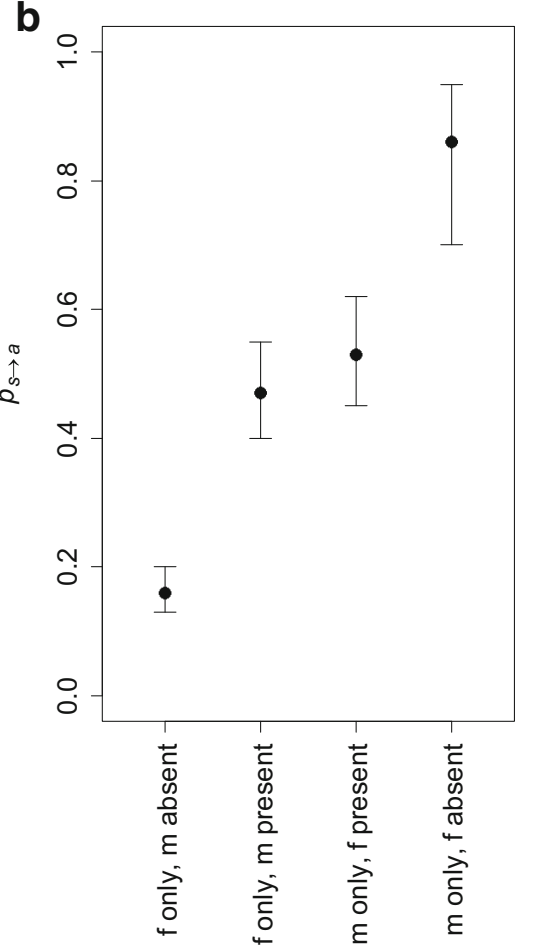

d

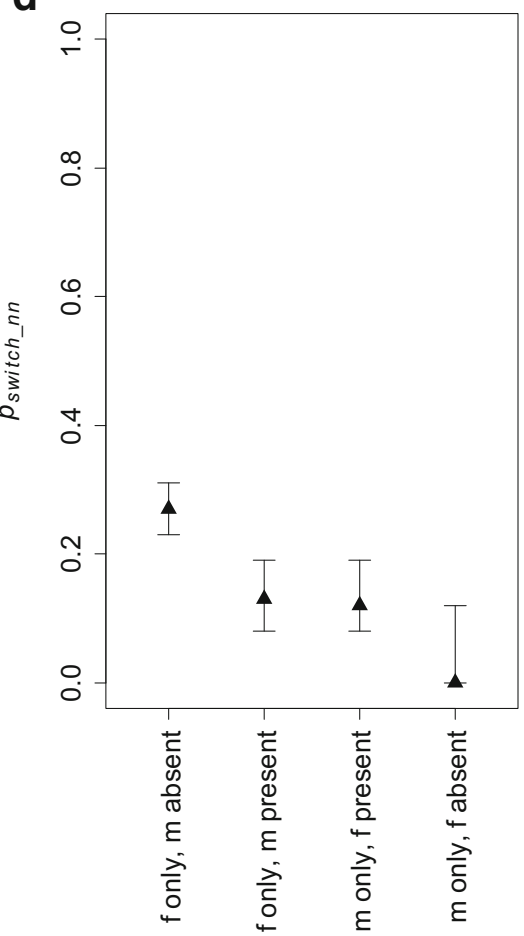

social dynamics might buffer changes in transmission processes (in fish populations) over a surprisingly large density range. The ability of social systems to self-stabilise is wellknown from social insects, where honey bees for example, maintain certain proportions of "in-door" and "out-door" workers (Huang et al. 1998; Schulz et al. 1998). In vertebrates, the robustness of social dynamics to external stimuli is less well documented.

Density independence has also been reported in the context of collective behaviour from starlings, Sturnus vulgaris (Ballerini et al. 2008). When flying in large flocks each individual was reported to follow a fixed number (six to 
Fig. 7 In pool 1a, five new females were introduced for three days. The probabilities (plus $95 \%$ confidence intervals) for unmanipulated pool (UM), day 1 , day 2, day 3 of the manipulation and the translocation (see Table 1) are shown for a ending contact with a specific neighbour ( $p_{\text {leave }}$ $\mathrm{nn})$, b ending social contact in general $\left(p_{s \rightarrow a}\right)$, $\mathbf{c}$ ending being alone $\left(p_{a \rightarrow s}\right)$, and $\mathbf{d}$ changing to a different social partner while staying social $\left(p_{\text {switch_nn }}\right)$ are shown for all pools before manipulation (see also Fig. 1 for the full Markov model) a

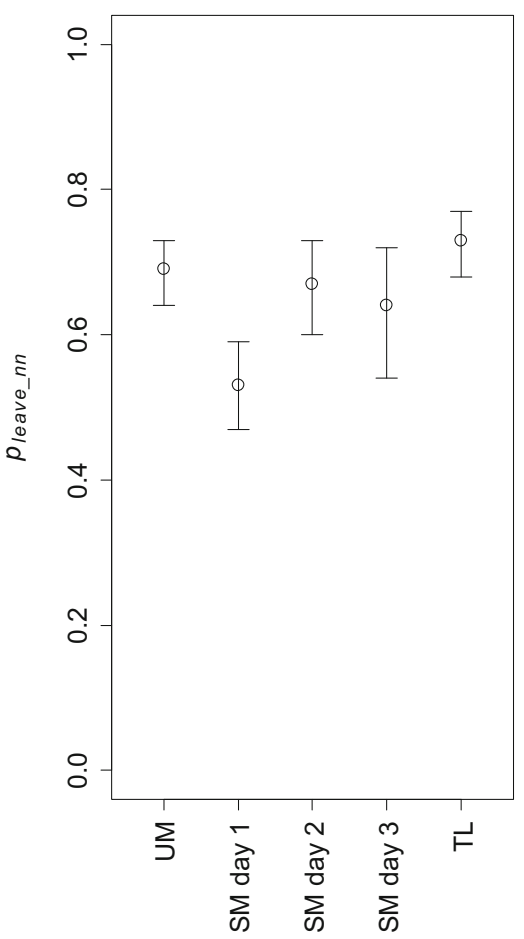

c

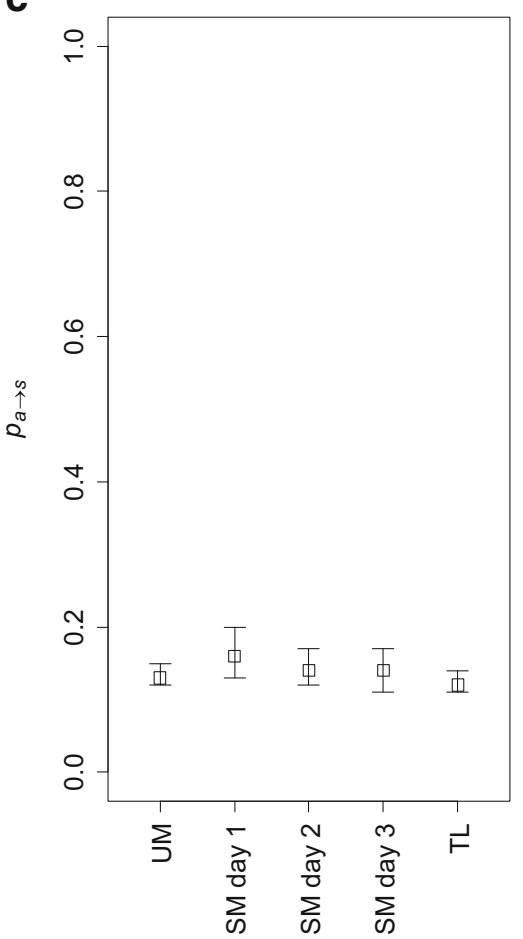

b

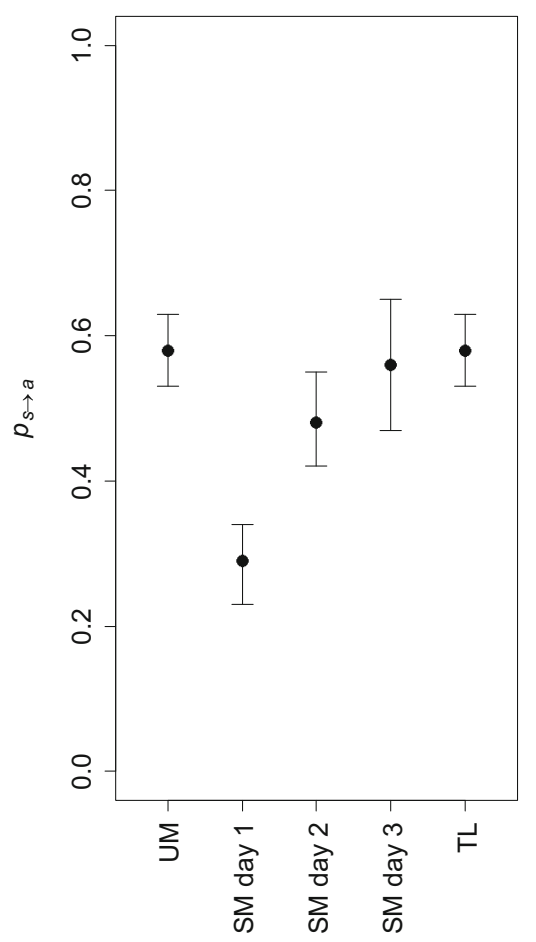

d

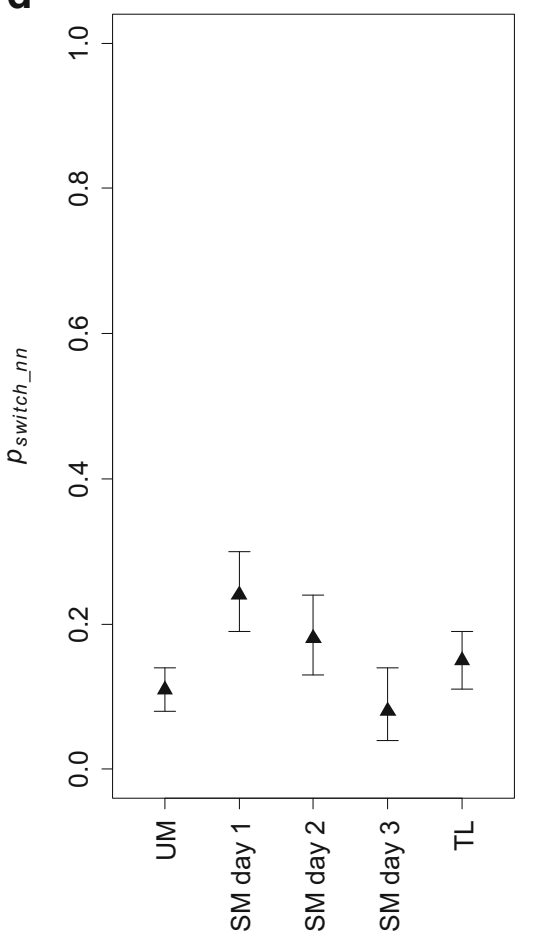

seven individuals) of nearest neighbours largely independently of flock density. Ballerini et al. (2008) argued that density-independent behaviour is suggestive of topological interaction dynamics. It remains to be explored whether this is also the case in guppies and in the wider context of fission-fusion behaviour.

\section{Social manipulations}

We observed a striking difference in male and female networks in response to the removal of the opposite sex. Females spent considerably more time being social whereas males maintained only a rudimentary social network. We did not 
study the medium- to long-term effects of such sex removals but the introduction of additional females in pool 1a showed that the system returned to its usual dynamics again relatively quickly. The interest of males in novel females is well documented (and not only for guppies, but also for other vertebrate species) (Fiorino et al. 1997; Kelley et al. 1999). However, in our study, it did not last for more than 1-2 days. Experiments in captivity showed that the presence of males disrupted female social behaviour and female preferences in guppies (Darden and Croft 2008). A study on captive catsharks, $S$. canulcula, showed that both the presence of new males and females can have a disruptive influence on existing female networks but that the effect of the introductions partly depended on the strength of the existing social ties between females (Jacoby et al. 2010). We found that the interaction duration between females was clearly shortened by the presence of males. However, interaction duration is not the parameter that is important for the expression of social preference in our system. Guppies use interaction frequency to express preferences and this was found to be unchanged in the presence/ absence of males. Differences in lab and field results are probably accounted for by the fact that in the field, females have much more space (familiar surroundings and a pool depth profile) to shake off harassing males.

In contrast to the presence of males, which is disruptive to social structure, the presence of females acted like a social "glue" without which the male-only network fragments. Presumably a network of male guppies only would soon collapse altogether with males emigrating from the pool in search of females.

\section{Evolution of fission-fusion dynamics}

An important (and maybe surprising) aspect of the social dynamics of our guppy system is the fact that it is well approximated by a geometric distribution (Fig. S1, see also Wilson et al. 2014) which raises questions regarding the evolutionary pressures that shaped this aspect of guppy biology. The vigilance patterns of some bird species are known to follow a negative exponential distribution (Bednekoff and Lima 2002), the only difference being that a geometric distribution is based on discrete time intervals. Bednekoff and Lima (2002) presented a compelling argument for the consideration of predator attack strategies in the context of animal vigilance patterns suggesting that regular vigilance patterns should be adopted if a predator appears at random (such as a hunting raptor which suddenly appears and immediately attacks) and variation in vigilance intervals should be favoured in the case of stalking predators (see also Scannell et al. 2001). We applied the same logic to the evolution of the fission-fusion dynamics observed in our system. If a predator is almost always present and monitors prey for moments when it is alone, as in the case with predators such as the pike cichlid Crenicichla frenata (the main guppy predator in Trinidad; Magurran 2005), then a geometric distribution could be adaptive because there would be no typical period for which the predator has to wait for the prey to be alone. Therefore a geometric distribution could be a shoaling strategy that is cognitively easy to implement (i.e. for every given time unit a fish makes a decision whether to be social or asocial with a fixed probability) but is also impossible for a predator to predict. The geometric distribution also has interesting implications for social preferences in guppies, which are not expressed in the duration of associations (which always follow a geometric distribution) but in the frequency with which particular individuals are selected as shoaling partners.

This brings us to the wider question of whether fissionfusion systems (which are common in nature, Krause and Ruxton 2002; Couzin 2006) are more generally selected to show geometric distributions because of similar selection pressures. Food competition is a common cost of group-living and might select for time spent alone whereas protection from predators is often a benefit of social life (Krause and Ruxton 2002). Little is known, however, regarding the dynamics of time periods spent alone and spent social; i.e. the resulting fissionfusion dynamics. Recent studies on the shoaling dynamics of other poeciliids (JK and D. Bierbach, unpublished work) and on juvenile lemon sharks, Negaprion brevirostris (Wilson et al. 2015), and bonefish, Albula vulpes (Wilson, unpublished work), shows that their behaviour also follows a geometric distribution. If the fission-fusion dynamics of prey populations that have certain types of predators is indeed selected to produce a geometric distribution, then this would have important repercussions for the evolution of social preferences.

Acknowledgments We thank Meint-Hilmar Broers and Jan Trebesch for help in the field. This study received funding from the Alexander von Humboldt Foundation (ADMW) and the BehaviourType project granted by the Gottfried-Wilhelm-Leibniz Association's Pact for Innovation and Research (JK). We would also like to thank Associate Editor Jan Lindström and two anonymous reviewers for their valuable input.

Conflict of interest The authors declare that they have no conflict of interest.

Ethical approval This research was performed in accordance with the laws, guidelines, and ethical standards of the country in which they were performed (Trinidad).

\section{References}

Ballerini M, Cabibbo N, Candelier R et al (2008) Empirical investigation of starling flocks: a benchmark study in collective animal behaviour. Anim Behav 76:201-215

Bednekoff PA, Lima SL (2002) Why are scanning patterns so variable? An overlooked question in the study of anti-predator vigilance. $\mathrm{J}$ Avian Biol 33:143-149

Blonder B, Wey TW, Dornhaus A, James R, Sih A (2012) Temporal dynamics and network analysis. Method Ecol Evol 3:958-972

Borner KK, Krause S, Mehner T, Uusi-Heikkilä S, Ramnarine IW, Krause J (2015). Turbidity affects social dynamics in Trinidadian 
guppies. Behav Ecol Sociobiol (published online, doi:10.1007/ s00265-015-1875-3).

Brierley AS (2014) Diel vertical migration. Curr Biol 24:R1074-R1076

Butts CT (2008) A relational event framework for social action. Sociol Methodol 38:155-200

Couzin ID (2006) Behavioral ecology: social organization in fissionfusion societies. Curr Biol 16:R169-R171

Croft DP, James R, Krause J (2008) Exploring animal social networks. Princeton University Press, Princeton

Croft DP, Krause J, Darden SK, Ramnarine IW, Faria JJ, James R (2009) Behavioural trait assortment in a social network: patterns and implications. Behav Ecol Sociobiol 63:1495-1503

Darden SK, Croft DP (2008) Male harassment drives females to alter habitat use and leads to segregation of the sexes. Biol Lett 4:449-451

Darden SK, James R, Ramnarine IW, Croft DP (2009) Social implications of the battle of the sexes: sexual harassment disrupts female sociality and social recognition. Proc R Soc Lond B 276:2651-2656

Drewe JA, Perkins SE (2014) Disease transmission in animal social networks. In: Krause J, James R, Franks DW, Croft DP (eds) Animal social networks. Oxford University Press, pp 95-110

Fiorino DF, Coury A, Philipps AG (1997) Dynamic changes in nucleus accumbens dopamine efflux during the Coolidge effect in male rats. J Neurosci 17:4849-4855

Flack JC, Girvan M, de Waal FBM, Krakauer DC (2006) Policing stabilizes construction of social niches in primates. Nature 439:426-429

Griffiths SW, Magurran AE (1997) Schooling preferences for familiar fish vary with group size in a wild guppy population. Proc R Soc Lond B 264:547-551

Hensor E, Couzin ID, James R, Krause J (2005) Modelling densitydependent fish shoal distributions in the laboratory and field. Oikos 110:344-352

Henzi SP, Lusseau D, Weingrill T, van Schaik CP, Barrett L (2009) Cyclicity in the structure of female baboon social networks. Behav Ecol Sociobiol 63:1015-1021

Huang ZY, Plettner E, Robinson GE (1998) Effects of social environment and worker mandibular glands on endocrine-mediated behavioral development in honey bees. J Comp Physiol A 183:143-152

Jacoby DMP, Busawon DS, Sims DW (2010) Sex and social networking: the influence of male presence on social structure of female shark groups. Behav Ecol 21:808-818

Kelley JL, Graves JA, Magurran AE (1999) Familiarity breeds contempt in guppies. Nature 401:661-662

Krause J, Ruxton GD (2002) Living in groups. Oxford University Press, Oxford

Krause J, Lusseau D, James R (2009) Animal social networks: an introduction. Behav Ecol Sociobiol 63:967-973

Krause J, James R, Franks DW, Croft DP (eds) (2014) Animal social networks. Oxford University Press, Oxford
Kurvers RHJM, Krause J, Croft DP, Wilson ADM, Wolf M (2014) Ecological and evolutionary consequences of social networks: emerging topics. Trends Ecol Evol 29:326-335

Magurran AE (2005) Evolutionary ecology: the Trinidadian guppy. Oxford University Press, Oxford

Makris NC, Ratilal P, Jagannathan S, Gong Z, Andrews M, Bertsatos I, Godo OR, Nero RW, Jech JM (2009) Critical population density triggers rapid formation of vast oceanic fish shoals. Science 323:1734-1737

Martin P, Bateson P (2007) Measuring behaviour: an introductory guide. Cambridge University Press, Cambridge

Newman MEJ (2003) The structure and function of complex networks. Siam Rev 45:167-256

Nightingale G, Boogert NJ, Laland KN, Hoppitt W (2014) Quantifying diffusion in social networks: a Bayesian approach. In: Krause J, James R, Franks DW, Croft DP (eds) Animal social networks. Oxford University Press, Oxford, pp 38-52

Pinter-Wollman N, Hobson EA, Smith JE et al (2013) The dynamics of animal social networks: analytical, conceptual, and theoretical advances. Behav Ecol 25:242-255

Rangeley RW, Kramer DL (1998) Density-dependent antipredator tactics and habitat selection in juvenile pollock. Ecology 79:943-952

Scannell J, Roberts G, Lazarus J (2001) Prey scan at random to evade observant predators. Proc R Soc Lond B 268:541-547

Schulz DJ, Huang ZY, Robinson GE (1998) Effects of colony food shortage on behavioral development in honey bees. Behav Ecol Sociobiol 42:95-303

Strier KB, Mendes SL (2012) The Northern muriqui (Brachyteles hypoxanthus): Lessons on behavioral plasticity and population dynamics from a critically endangered species. In: Kappeler PM, Watts DP (eds) Long-term field studies of primates. Springer-Verlag, Berlin, pp 125-140

Tranmer M, Marcum CS, Morton FB, Croft DP, de Kort SR (2015) Using the relational event model (REM) to inesvitgate the temporal dynamics of animal social networks. Anim Behav 101:99-105

Wey TW, Burger JR, Ebensperger LA, Hayes LD (2013) Reproductive correlates of social network variation in plural breeding degus (Octodon degus). Anim Behav 85:1407-1414

Wilson ADM, Krause S, James R, Croft DP, Ramnarine IW, Borner KK, Clement RJG, Krause J (2014) Dynamic social networks in guppies (Poecilia reticulata). Behav Ecol Sociobiol 68:915-925

Wilson ADM, Brownscombe JW, Krause J, Krause S, Gutowsky LFG, Brooks EJ, Cooke SJ (2015) Integrating network analysis, sensor tags and observation to understand shark ecology and behaviour. Behav Ecol. doi:10.1093/beheco/arv115

Wittemyer G, Douglas-Hamilton I, Getz WM (2005) The socioecology of elephants: analysis of the processes creating multitiered social structures. Anim Behav 69:1357-1371 